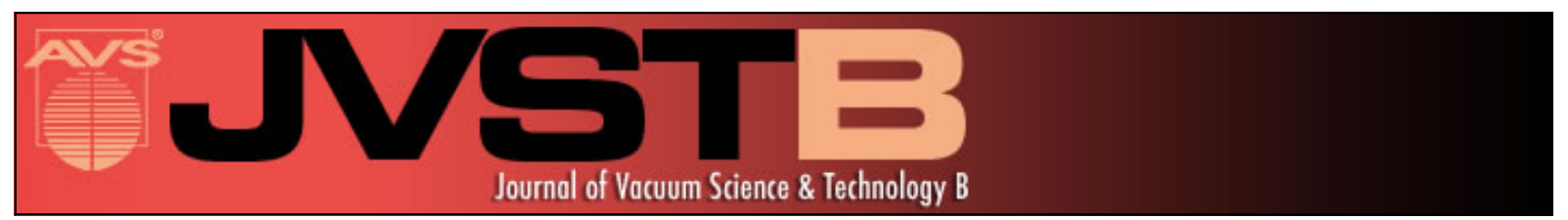

\title{
Topography-free sample for thermal spatial response measurement of scanning thermal microscopy
}

Yunfei Ge, Yuan Zhang, Jonathan M. R. Weaver, Haiping Zhou, and Phillip S. Dobson

Citation: Journal of Vacuum Science \& Technology B 33, 06FA03 (2015); doi: 10.1116/1.4933172

View online: http://dx.doi.org/10.1116/1.4933172

View Table of Contents: http://scitation.aip.org/content/avs/journal/jvstb/33/6?ver=pdfcov

Published by the AVS: Science \& Technology of Materials, Interfaces, and Processing

\section{Articles you may be interested in}

The influence of the substrate thermal conductivity on scanning thermochemical lithography

J. Appl. Phys. 111, 124317 (2012); 10.1063/1.4729809

High-resolution scanning thermal probe with servocontrolled interface circuit for microcalorimetry and other applications

Rev. Sci. Instrum. 75, 1222 (2004); 10.1063/1.1711153

High spatial resolution thermal conductivity of bulk $\mathrm{ZnO}(0001)$

J. Appl. Phys. 91, 890 (2002); 10.1063/1.1426234

Simulation and correction of geometric distortions in scanning Kelvin probe microscopy

J. Vac. Sci. Technol. A 18, 1051 (2000); 10.1116/1.582472

High-contrast topography-free sample for near-field optical microscopy

Appl. Phys. Lett. 76, 1206 (2000); 10.1063/1.125984

\section{HIDEN}

\section{Instruments for Advanced Science} w www.HidenAnalytical.com E info@hiden.co.uk CLICK TO VIEW our product catalogue

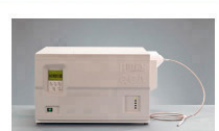

Gas Analysis

dynamic measurement of reaction gas steans catalysis and thermal analysis molecular beam studies

, dissolved species probes

fermentation environmental and ecological studies

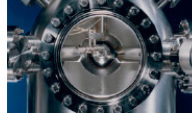

Surface Science

UHVTPD

SIMS emental imaging - surface mapping

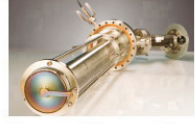

Plasma Diagnostics plasma source characterization etch and deposition process reaction , analysis of neutral and radical species

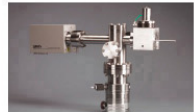

Vacuum Analysis , partial pressure measurement and contro of process gases reactive sputter process contro vacuum diagnostics

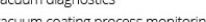




\title{
Topography-free sample for thermal spatial response measurement of scanning thermal microscopy
}

\author{
Yunfei Ge, ${ }^{\text {a) }}$ Yuan Zhang, Jonathan M. R. Weaver, Haiping Zhou, and Phillip S. Dobson \\ School of Engineering, University of Glasgow, Rankine Building, Oakfield Avenue, Glasgow G12 8LT, \\ United Kingdom
}

(Received 22 June 2015; accepted 2 October 2015; published 13 October 2015)

\begin{abstract}
A novel fabrication technique is described for the production of multimaterial, lithographically defined, topography-free samples for use in experiments to investigate the nature of contrast in scanning probe microscopy (SPM). The approach uses a flat sacrificial substrate as the base for fabrication, which is deleted in the final step. This leaves an exposed, flat surface with patterns of materials contrast defined during the lithography stages. In the example application presented, these are designed to challenge the detection ability of a scanning thermal microscopy (SThM) probe, although many other applications can be envisioned. There are many instances in SPM where images can exhibit topographically induced artifacts. In SThM, these can result in a change of the thermal signal which can easily be misinterpreted as changes in the sample thermal conductivity or temperature. The elimination of these artifacts through postprocessing requires a knowledge of how the probe responds thermal features of differing sizes. The complete sample fabrication process, followed by successful topographic/thermal scanning is demonstrated, showing sub- $1.5 \mathrm{~nm}$ topography with a clear artifact-free thermal signal from sub-100 nm gold wires. The thermal spatial resolution is determined for the sample materials and probe used in this study to be in the range of 35-75 nm. (C) 2015 American Vacuum Society. [http://dx.doi.org/10.1116/1.4933172]
\end{abstract}

\section{INTRODUCTION}

As research and manufacturing processes continue to push electronic and optical devices further into the nanoscale, the measurement of such small structures has become ever more challenging for conventional metrology techniques. Scanning probe microscopy (SPM), with its ability to study surfaces in various environments with nanoscale resolution, has become a popular tool. The ability to integrate various sensors directly onto the SPM tip enables the mapping of many physical characteristics beyond topography, such as electrical conductivity, ${ }^{1}$ magnetic domain structures, ${ }^{2}$ and temperature. ${ }^{3}$ However, complex tip-sample interactions can make it difficult to obtain truly quantitative results from either contact or noncontact SPM. A general problem is that artifacts caused by topographic variations of the sample can easily obscure contrast resulting from changes in the properties of a material. Many SPM techniques have been demonstrated to suffer from severe topographically induced artifacts, including conductive AFM (Ref. 4) and scanning near-field optical microscopy. ${ }^{5-7}$ In order to fully investigate the interactions between tip and sample, it is essential to obtain SPM/sensor data from samples without significant topography. Here, we present a reliable method for the production of lithographically patterned samples, exhibiting strong material contrast but with minimal surface topography. The utility of these samples is then demonstrated using scanning thermal microscopy (SThM) in active mode. This mode employs a sensor, located at the AFM tip, which has been heated above ambient temperatures through Joule heating. As the tip is scanned

$\overline{{ }^{a} \text { Electronic mail: y.ge.1@research.gla.ac.uk }}$ across a surface, changes in tip-sample thermal interaction result in variations in tip temperature, which are detected electrically.

Thermal measurement at the nanoscale is a subject of significant practical importance. Temperature control has become perhaps the most crucial issue in nanoscale devices due to Joule heating caused by very high power densities. ${ }^{8}$ This issue may be further exacerbated in advanced devices such as nanowire-based semiconductor transistors as a result of their low thermal conductivity. ${ }^{9-11}$ A number of techniques, such as Raman spectroscopy, ${ }^{12}$ infrared imaging, ${ }^{13}$ and near-field optical thermography, ${ }^{14}$ have already been developed to measure the temperature in microdevices. However, the resolution of these techniques is limited to the micrometer-scale and is not suitable for obtaining temperature distributions in sub-100 $\mathrm{nm}$ devices. SThM ${ }^{15-17}$ makes the achievement of high spatial resolution temperature measurement a reality. Besides temperature measurement, the nanoscale study of material thermal properties such as thermal conductivity can be achieved using active mode SThM. This has been an area of focus in recent years, offering many applications in microelectronics where functional materials such as strained layers may not be available as bulk samples for measurement. ${ }^{18-20}$ However, as with many other SPM techniques, SThM suffers from topographically induced artifacts in the data it generates. To thoroughly interpret the results obtained using SThM, these artifacts have to be either eliminated or be comprehensively understood. ${ }^{21-23}$

SThM thermal data distortion, induced by heat transfer between the cantilever and the sample through the air, is considered to be one of the major artifacts. However, advanced scanning methods such as the "double-scan technique" 24 and "topography-correction technique" ${ }^{23}$ have been demonstrated 
to effectively diminish this artifact. Another major artifact, induced by the topography of the sample, is hard to eliminate due to its complexity ${ }^{25-28}$ and it is this example that will be considered here. The heat transfer between the probe tip and the sample has been proven to occur through a combination of pathways, including thermal conduction through the solid-solid contact, water meniscus, and air and thermal radiation (in near and far fields) when performing measurement under ambient conditions. Gotsmann and Lantz ${ }^{29}$ have studied the effect of surface roughness on the thermal interface conductance under vacuum conditions. By constructing a model that describes the thermal transport across individual contact points, they defined the conductance per atom-atom contact as

$$
G_{\text {atom }}=G_{Q} N \tau,
$$

where $G_{Q}$ is the universal thermal conductance of a quantum point contact, $N$ is the number of different phonon modes, and $\tau$ is the corresponding transmission coefficient. As the number of contact points varied with changes in surface roughness or topography, the thermal signal was seen to vary due to the change in the number of atom-atom contacts. Several groups have observed this phenomenon in their thermal scans, for example, Soudi et al. ${ }^{21}$ reported a "double-line-like" thermal profile when scanning 40-60 nm heated nanowires in both air and vacuum in passive mode. In the presence of these topographically induced artifacts, the local temperature of the nanowire could only be approximated by averaging the whole region, which is undoubtedly inaccurate.

Efforts have been made to obtain real thermal images without topographically induced artifacts by employing an algorithm to postprocess the thermal signal. ${ }^{30}$ However, this approach is not straightforward for samples which exhibit both material and topographic variation. In summary, knowledge of the thermal spatial resolution of any given SThM probe type will benefit the quantitative study of heat transfer mechanisms between the tip and sample, which is still a controversial subject. ${ }^{31}$ Recent work in this area has focused on ways to estimate the real resolution while taking into account the meniscus radius and issues relating to the mechanical contact. $^{24,32-34}$

In contrast to other SThM probes, such as the Wollaston probe which exhibits poor lateral resolution, $\sim 1.5 \mu \mathrm{m}$, due to a blunt tip, ${ }^{31,35}$ microfabricated probes have a nanoscale tip radius allowing them to image smaller thermal features. ${ }^{25}$ SThM probes are used in two modes-active mode in which the self-heated probe is cooled by transferring heat to the sample and passive mode in which the probe receives heat flowing from the heated sample. Zhou et al. ${ }^{36}$ observed a thermal spatial resolution of hundreds of nanometers by scanning a $300-1500 \mathrm{~nm}$ thick palladium acetate thin film with a probe operating $20-40^{\circ} \mathrm{C}$ above room temperature. In addition, Sadat et $a l .{ }^{37}$ used a thermocouple tip with $\sim 20 \mathrm{~nm}$ radius to scan heated features on a metallic surface, successfully achieving sub- $100 \mathrm{~nm}$ thermal-spatial resolution. Shi et $a l^{38}$ scanned a heated $14 \mathrm{~nm}$ diameter multiwall carbon nanotube and observed a resolution of around $50 \mathrm{~nm}$, consistent with the tip radius of the SThM probe used. Hinz et al. ${ }^{39}$ observed $25 \mathrm{~nm}$ thermal-spatial resolution under high vacuum conditions in experiments aimed at determining the thermal conductivity of a $3 \mathrm{~nm}$ thick $\mathrm{HfO}_{2}$ film. However, few researchers have considered the effect of topography on the measurement other than to note its presence.

In this paper, we present a fabrication method for the production of a topography-free sample consisting of two materials, gold and $\mathrm{Si}_{3} \mathrm{~N}_{4}$, with a large difference in thermal conductivity. Thin-film narrow gold wires have been patterned and then scanned using a microfabricated SThM probe working in DC active mode in air. By analyzing the results, the thermal spatial resolution of the probe in the absence of topographic artifacts can be clearly observed.

\section{DESIGN AND FABRICATION}

When considering sample planarization, chemical mechanical planarization (CMP), which is widely used in the integrated circuit industry is an obvious choice. However, this technique has drawbacks such as dishing and erosion, where some regions and materials polish faster than others limiting the range of different materials within can be used. This is combined with the high complexity and cost of developing a process and the potential for significant surface damage, making CMP unattractive for scanning technology. ${ }^{40}$ An alternative approach, employing beam exit Argon ion cross-section polishing has been proposed by Kolosov et al. ${ }^{41}$ This provides a method to produce a sample surface with topography down to $1 \mathrm{~nm}$, which is suitable for SPM scanning. However, this approach offers limited flexibility in the definition of material patterns, as well as limited control of the feature thickness.

One possible approach for eliminating surface topography of a lithographic pattern is through filling an etched cavity using a second material. This would require two aligned levels of lithography for a single pattern: one for etching and the other for deposition. However, there will be inevitable misalignment between the levels, even if the most accurate alignment methods were employed. ${ }^{42}$ The consequence of any misalignment would be inconsistent thickness resulting from a step or trench between the cavity and the deposited material, resulting in an overall increase in peak-to-peak topographic variation.

The sample fabricated in this work employed a different approach. The process is based around common, generic resist-based pattern transfer techniques compatible with a wide range of materials: lithographically defined metal patterns were fabricated on a sacrificial substrate before being embedded into an epoxy-supported backing. Finally, the initial "sacrificial" substrate was deleted. This method takes advantage of the high quality surfaces obtainable using CMP polished wafers, while simultaneously avoiding the chemical and mechanical damage generated by polishing the pattern itself. 
The complete fabrication process flow is shown in Fig. 1. The desired pattern is deposited onto a sacrificial GaAs substrate as shown in step (a). This process employs a traditional bilayer PMMA resist patterned using $100 \mathrm{kV}$ electron beam lithography. ${ }^{43}$ The resist stack consisted of $38 \mathrm{~nm}$ of PMMA having a molecular weight of $85 \mathrm{kDa}$ and $38 \mathrm{~nm}$ of PMMA having a molecular weight of $350 \mathrm{kDa}$. Twenty nanometers gold was evaporated, followed by "lift-off" in $50{ }^{\circ} \mathrm{C}$ acetone for $12 \mathrm{~h}$. The pattern consisted of wires located in a $1 \mu \mathrm{m}$ gap between two large gold pads, permitting easy pattern location within the AFM/SThM using an optical microscope. It should be noted that the process is compatible with thicker layers of material if the highest resolution is not required and if thicker resist is used. Structures of thickness up to $150 \mathrm{~nm}$ have been successfully patterned by the authors using this technique.

The fabrication of the backing substrate is a multilayer process as shown in the following steps from Fig. 1: $400 \mathrm{~nm}$ low stress inductively coupled plasma chemical vapor deposition (ICP-CVD) $\mathrm{SiN}_{\mathrm{x}}$ was deposited ${ }^{44}$ onto the sample which had been dehydrated on $180^{\circ} \mathrm{C}$ hot plate for $5 \mathrm{~min}$ to improve adhesion (b); then a $100 \mu \mathrm{m}$ thick layer of SU-8 3050 was spun onto the processed face of the sample (c); and finally, the sample was heated and a piece of quartz was placed onto the hot SU-8 to provide a rigid mechanical handle for the released sample (d).

Once the full material stack was complete, the GaAs substrate was deleted using a citric acid/ $\mathrm{H}_{2} \mathrm{O}_{2}$ wet etch, uncovering the $\mathrm{Au}$ pattern and $\mathrm{SiN}_{\mathrm{x}}$ background. The finished sample is as shown in step (e).
GaAs was chosen as the substrate due to its availability as a polished wafer, electrical conductivity (useful when using electron-beam lithography) and relative ease of wet etching. ${ }^{45}$ Although silicon would appear to be a more economic choice, the available wet ${ }^{46}$ and dry etches ${ }^{47}$ would potentially damage the $\mathrm{Au} / \mathrm{SiN}_{\mathrm{x}}$ pattern resulting in a roughening of the surface. Wet etching of GaAs is possible using various solutions such as citric acid, $\mathrm{H}_{2} \mathrm{SO}_{4}, \mathrm{HCl}$, and $\mathrm{HF}$ with $\mathrm{H}_{2} \mathrm{O}_{2}$, but it was decided to use $50 \%$ citric acid with $\mathrm{H}_{2} \mathrm{O}_{2}$ to minimize pattern damage. ${ }^{48}$ The GaAs etch rate is strongly dependent on the temperature and ratio of citric acid and $\mathrm{H}_{2} \mathrm{O}_{2}$, with 5:1 citric acid: $\mathrm{H}_{2} \mathrm{O}_{2}$ giving the highest etch rate $(\sim 250 \mathrm{~nm} / \mathrm{min})$ at room temperature.

Gold was chosen as an evaporated pattern material due to its ease of fabrication, chemical resistance to etching, ${ }^{48}$ and high thermal conductivity. The thermal spatial resolution of SThM probes is commonly claimed to be sub-100 nm, with the heat conduction area considered to be the same as the tip curvature radius. ${ }^{33,49}$ To address these length scales, narrow gold wires with width ranging from 35 to $75 \mathrm{~nm}$ were fabricated as patterns.

The fabricated backing substrate is a sandwich structure: a conformal layer of $\mathrm{SiN}_{\mathrm{x}}$ around the gold structure, a thick layer of SU-8 cured epoxy and a rigid quartz handle. The $\mathrm{SiN}_{\mathrm{x}}$ offers three important properties: good adhesion to the gold patterns, large thermal contrast with gold, and resistance to the GaAs wet etch. The stack adopted replaces an initial design for the backing substrate which was simply to employ a double-layer of SU-8 3050. However, after release etching, few patterns were successfully transferred onto the
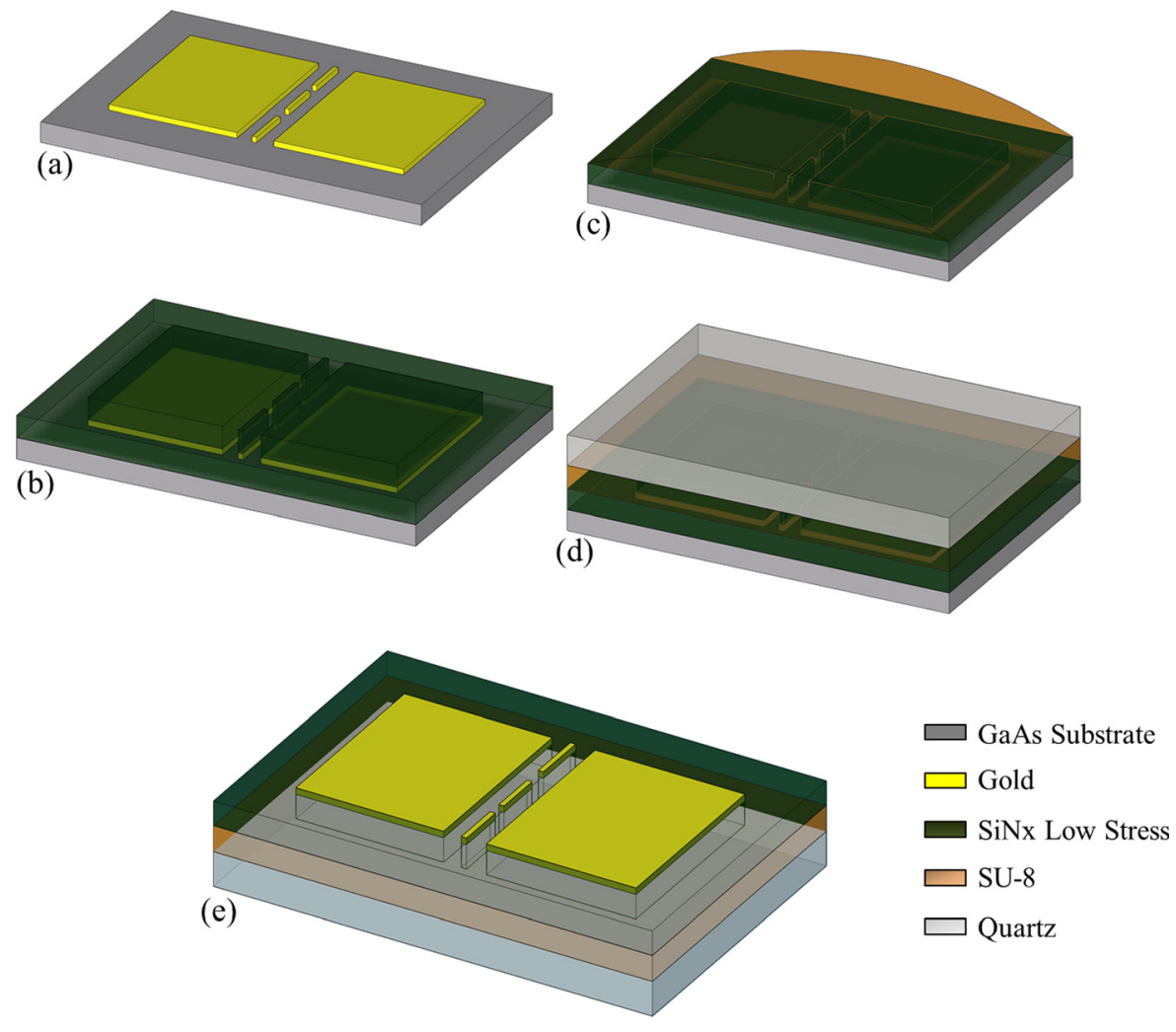

FIG. 1. (Color online) Fabrication process of the topography-free sample. 
SU-8 substrate due to the poor adhesion of gold to SU-8. ICP CVD was chosen due to its ability to deposit $\operatorname{SiN}_{x}$ at room temperature, maximizing the possible number of compatible materials for the lithographic pattern and eliminating any topography associated with differential thermal expansion during fabrication. Two types of $\mathrm{SiN}_{\mathrm{x}}$, compressive low stress $(\sim 100 \mathrm{MPa})$ and compressive high stress $(\sim 3 \mathrm{GPa})^{44}$ were tested. The deposition gas used for both is $\mathrm{SiH}_{4} / \mathrm{N}_{2}$ with flow rate of $7 / 6 \mathrm{sccm}$ under $4 \mathrm{mTorr}$ pressure. Both high stress and low stress $\mathrm{SiN}_{\mathrm{x}}$ provide an obvious improvement in the pattern adhesion, however, high stress $\operatorname{SiN}_{\mathrm{x}}$ induced small wrinkles in the Au pattern. Figure 2 shows optical and topographic AFM images of the sample after release etching. The wrinkles caused by the high stress $\mathrm{SiN}_{\mathrm{x}}$ are clearly visibly, particularly in the AFM image [Fig. 2(b)]. As a consequence further samples were prepared using low stress $\mathrm{SiN}_{\mathrm{x}}$ only.

The SU8 layer alone does not provide a flat backing material due to its tendency to bow after the GaAs substrate has been dissolved. Therefore, a piece of quartz, which is transparent to UV light, was used to cover the SU-8 at the end of the softbake. In the final structure, the SU8 is utilized more as an adhesive with low thermal conductivity than a substrate.

Following removal of the GaAs, the freshly exposed surface topography was checked using AFM. During this
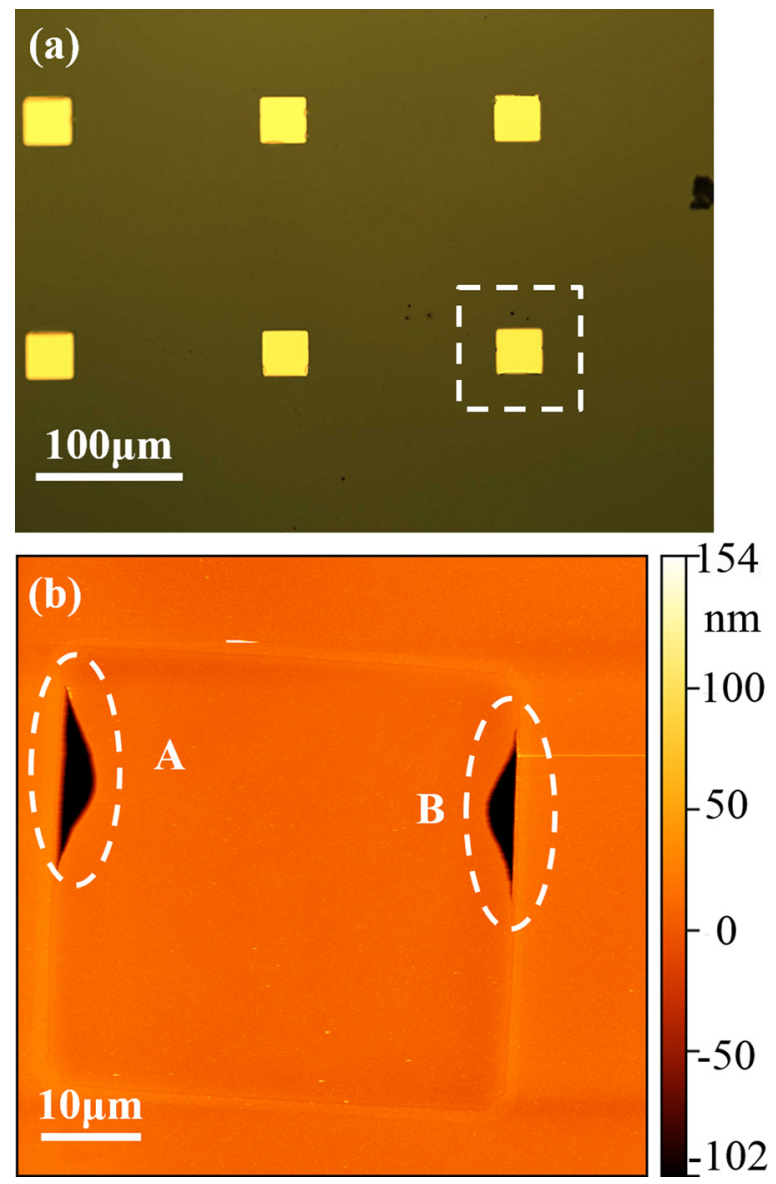

FIG. 2. (Color online) (a) Optical microscope image of the topography-free sample with high stress SiNx adhesion enhancement layer and (b) AFM height image of the same sample, the scan region is indicated by the dashed square in (a). Wrinkles are highlighted in regions A and B. inspection, a dependence of the residual topography on the thickness of $\mathrm{SiN}_{\mathrm{x}}$ was observed. Repeated experiments were used to optimize the $\mathrm{SiN}_{\mathrm{x}}$ thickness. Samples with 100-800 nm thick low stress $\mathrm{SiN}_{\mathrm{x}}$, deposited under the conditions previously described, were studied. For each sample, the average step height between the gold pattern and the $\mathrm{SiN}_{\mathrm{x}}$ was obtained by averaging the data from eight to ten patterns on each sample, as shown in Fig. 3. It can be seen that topography reduces with the increasing thickness of $\mathrm{SiN}_{\mathrm{x}}$, until no further improvement is observed $(400 \mathrm{~nm}$ and above). This is tentatively ascribed to the increased rigidity of the $\mathrm{SiN}_{\mathrm{x}}$ film with increasing thickness.

Figure 4 shows SEM and AFM images of the same region on the sample after completion. The images show $60 \mathrm{~nm}$ gold narrow wires fabricated at the center of the gap formed by two gold pads.

\section{EXPERIMENTAL SETUP}

The topography-free sample was scanned using microfabricated, thermally compensated cantilever probes developed in Glasgow. ${ }^{50}$ The experimental setup is shown in Fig. 5 with the inset showing an SEM micrograph of the probe. The probe is Joule-heated by a $0-2.0 \mathrm{~mA}$ DC current source controlled by a variable voltage reference, $\mathrm{V}$. The probe forms part of a Wheatstone bridge with $\mathrm{R}_{1}, \mathrm{R}_{3}$, and $\mathrm{R}_{4}$, enabling its resistance to be continuously monitored. The differential voltage from the bridge is amplified by an instrumentation amplifier with a voltage gain of 2000. Previous testing has shown that the probe can survive a maximum 2.5 mA DC current without a permanent change in its physical character.

The probe was calibrated using the experimental setup shown in Fig. 6(a). The SThM probe was immersed in an inert fluorocarbon liquid (flutec pp3, F2 Chemicals Ltd.) together with a PT100 thermal sensing element attached. The beaker was coupled to a Peltier thermoelectric module using thermal paste. The temperature of the Peltier was

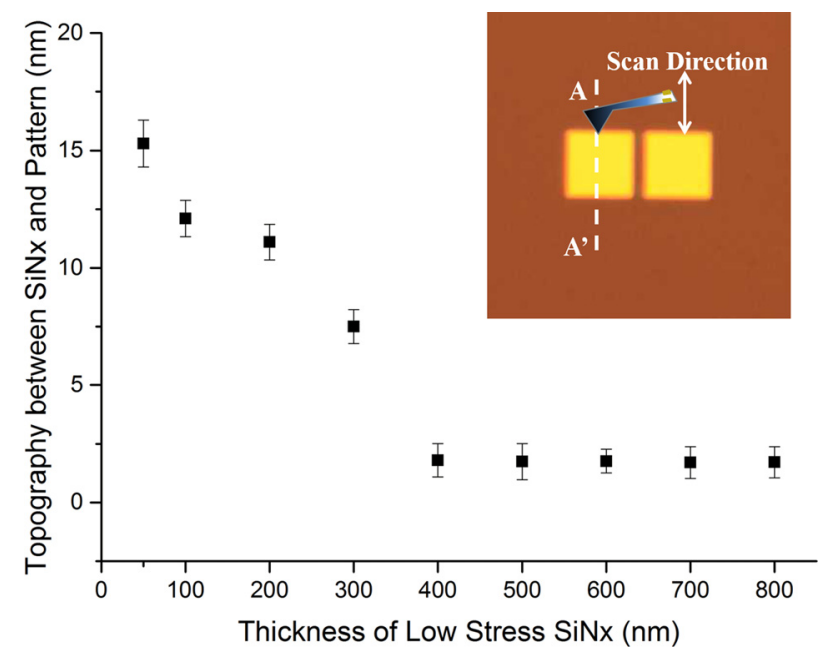

FIG. 3. (Color online) Step heights between gold and $\mathrm{SiN}_{\mathrm{x}}$ on the sample surface after release etch vs the thickness of $\operatorname{SiN}_{\mathrm{x}}$. The inset image shows the AFM scan used for obtaining the step height. 

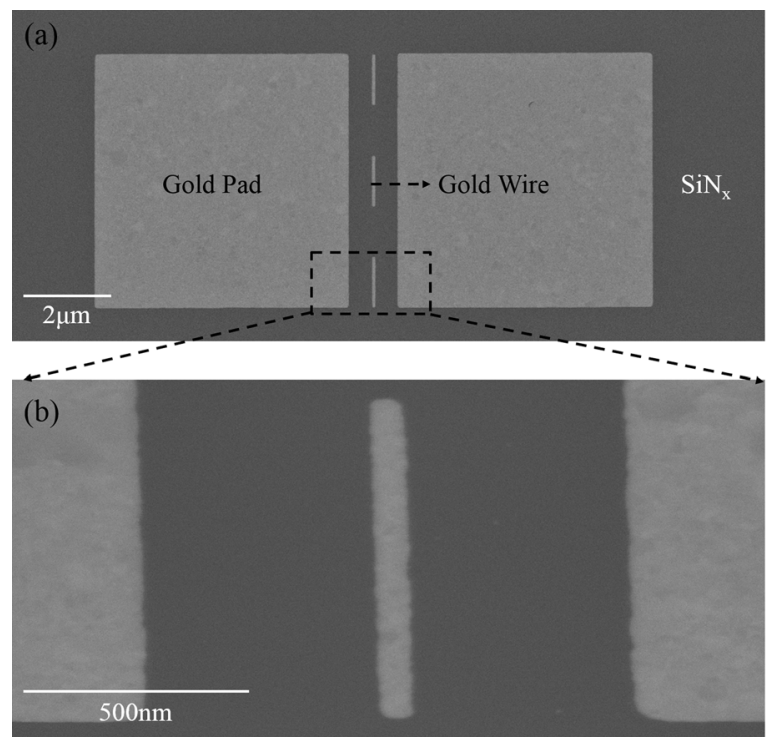
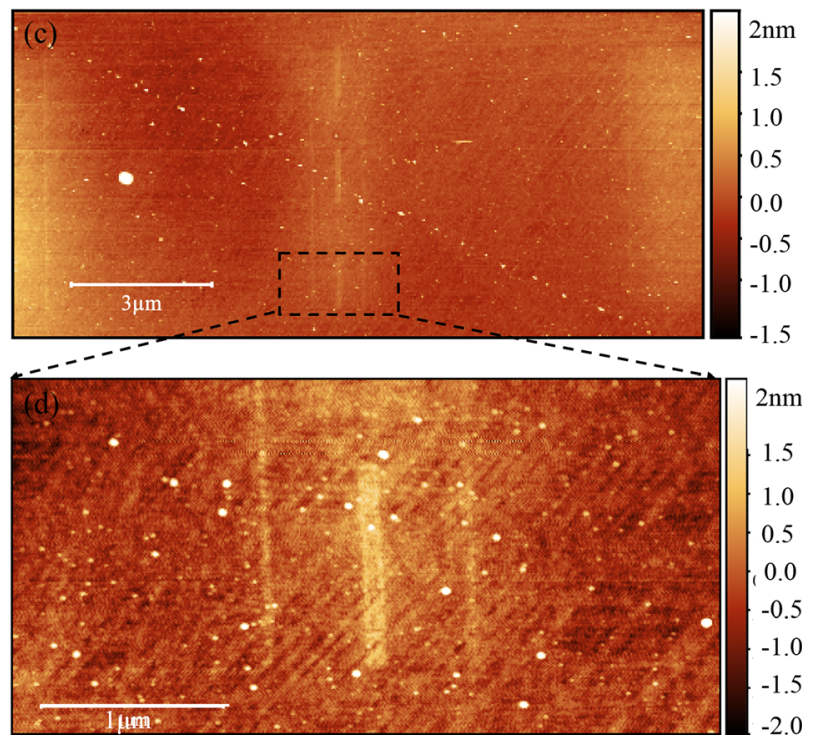

FIG. 4. (Color online) (a) SEM image of the pattern after release etching, (b) detail of the narrow wire zoomed in from (a), (c) tapping mode AFM topographic image showing the same area as (a), and (d) detail of the narrow wire zoomed in from (c) showing a residual topographic variation of less than 2 nm.

varied, and the four terminal resistance of the probe was measured using a multimeter. The temperature of the flutec pp3 was measured using the attached Pt100 resistance element, and it was assumed that this was the same temperature as the SThM probe. Ten values of the probe resistance and flutec temperature were recorded and are shown in Fig. 6(b). A least squares method was used to fit the data to a straight line, giving a sensitivity for the whole probe, consisting gold wires, pads, and the platinum tip of $3.14 \pm 0.02948 \mathrm{~K} / \Omega$. The cross sectional area of the platinum tip resistor is much smaller than that of the gold wires or pads on the probe. Therefore, $\sim 99 \%$ resistance of the whole probe is contributed by the platinum, ensuring a high sensitivity to the temperature change at the tip.

In all experiments, $0.8 \mathrm{~mA}$ was chosen as the bias current, resulting an out-of-contact probe effective temperature of $\sim 55^{\circ} \mathrm{C}$. This provided an appropriate compromise between a high thermal signal-to-noise ratio (SNR) with acceptable residual cantilever thermal bending.

\section{RESULTS AND DISCUSSION}

Figure 7 shows the SEM, AFM, and in-air SThM scans obtained from the same narrow wire. The width of the gold

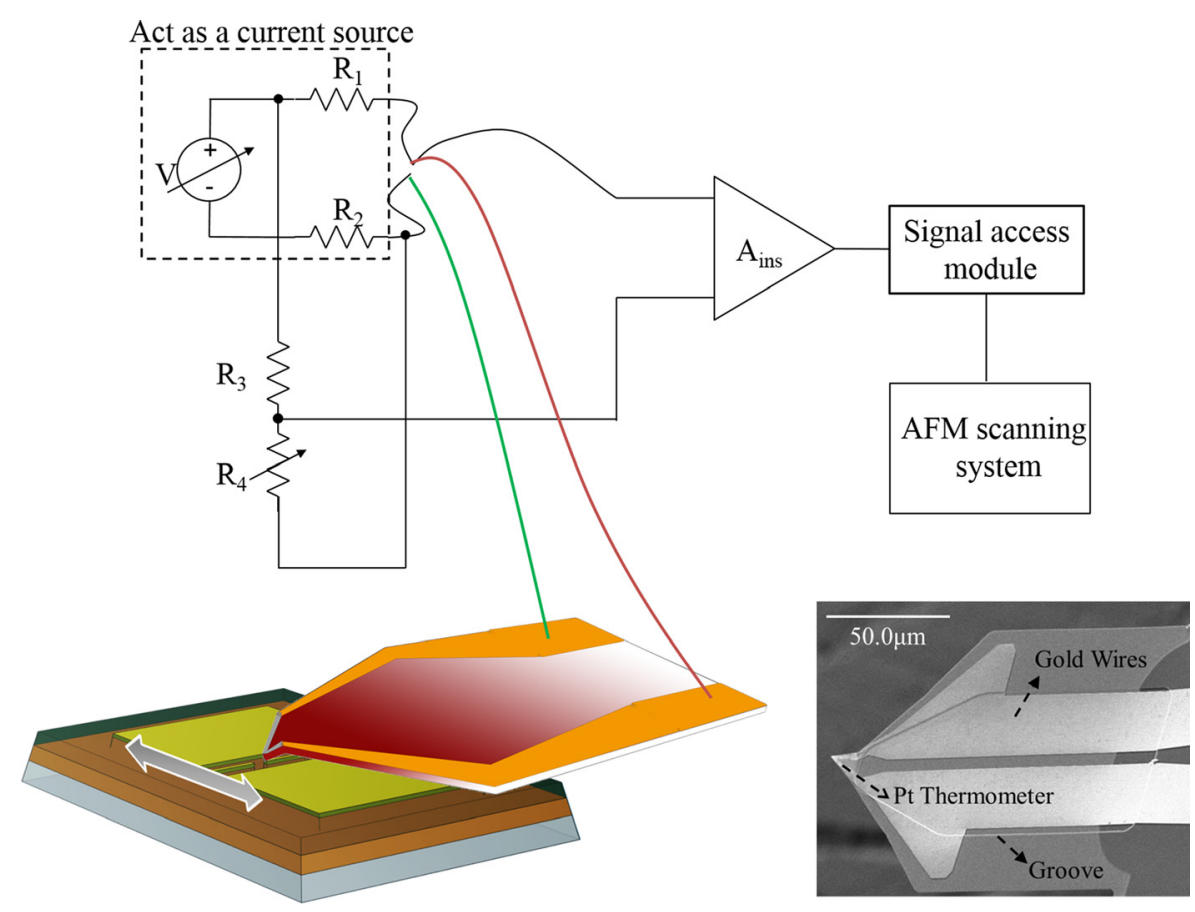

FIG. 5. (Color online) Schematic diagrams showing the electrical arrangement used to bias the probe and to detect the probe electrical resistance change. Inset image shows the SThM probe used with tip, Pt resistor, gold connecting wires and thermal compensation groove are all clearly visible. 


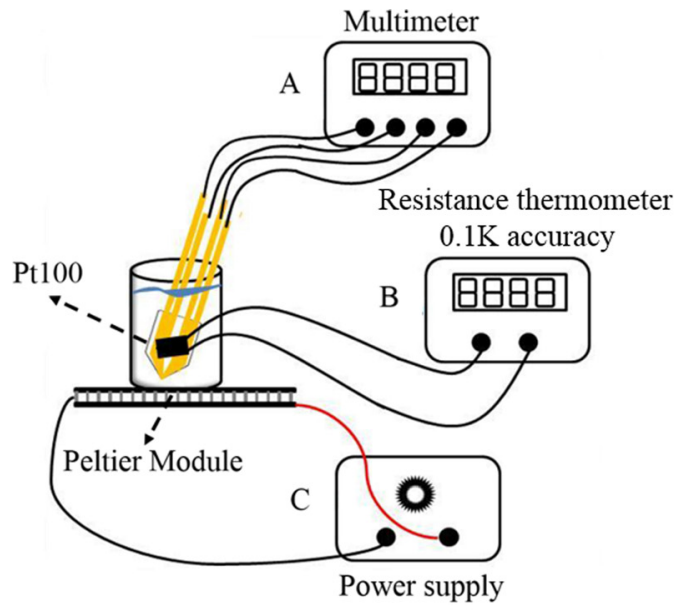

(a)

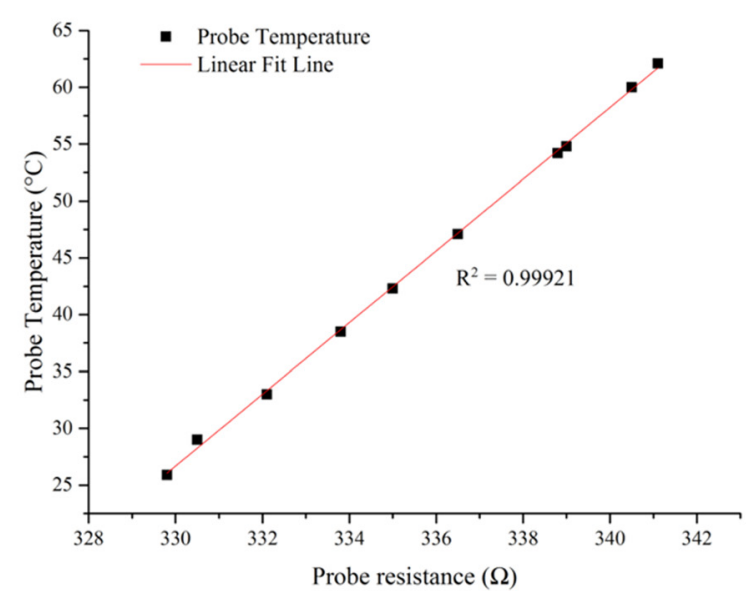

(b)

FIG. 6. (Color online) (a) Experimental setup for measuring the temperature coefficient of resistance of the probe, and (b) plot of the probe temperature vs the probe resistance. $\mathrm{R}^{2}$, ranging from 0 to 1 , is the coefficient of determination that indicates the linearity of the fitted line.

wire is $\sim 35 \mathrm{~nm}$ as determined by low vacuum backscatter SEM, avoiding the well-known errors associated with secondary electron scattering for linewidth measurement ${ }^{51}[$ Fig. 7(a)]. The topographic AFM image [Fig. 7(b)] and the thermal image [Fig. 7(c)] were obtained simultaneously during the thermal scan, and the $35 \mathrm{~nm}$ gold wire can be clearly seen. Data extracted from line traces at the same position on all images are plotted in Figs. 7(d) and 7(e). The step height associated with the $35 \mathrm{~nm}$ Au wire is $\sim 1.5 \mathrm{~nm}$, and the feature is clearly visible on the thermal image.

A comparison of the thermal signal intensity from the $35 \mathrm{~nm}$ wire with that of the gold pads shows that the magnitude of the thermal signal from the wire is lower than that of the gold pads, suggesting that it is "incompletely resolved."

This incomplete resolution can be attributed to the complex heat transfer mechanisms at the tip-sample contact. One powerful approach to aid understanding of the underlying heat transfer mechanisms between the tip and the sample is to use a thermal resistance network, as suggested by Luo et $a l .{ }^{52}$ Figure 8(a) summarizes the thermal resistance network for interactions between the probe and the sample. $R_{t}$ and $R_{s}$ represent the thermal resistance of the tip and the sample, respectively. The contact thermal resistance, $R_{\text {int }}$, represents thermal interaction between the tip and the sample and is a combination of many factors; however, solid to solid contact $\left(R_{s s}\right)$, water meniscus $\left(R_{w}\right)$, and air $\left(R_{\text {air }}\right)$ conduction are generally believed to dominate over heat transfer through radiation $\left(R_{\text {rad }}\right){ }^{28,33,53}$ Gorbunov et al. ${ }^{54}$ proved that heat dissipation to the sample from a heated tip is a function of the tip-sample contact radius as well as the sample materials being scanned. This has been investigated by Gotsmann ${ }^{29}$ who indicated that the contact thermal conductance is dependent on the pressure when the contact radius is constant. Hence, the tip-sample force was kept $20 \mathrm{nN}$ during our experiment. These thermal interactions have been recently discussed in detail by King et al. ${ }^{55}$ and Gomès et $a l .{ }^{28}$ As the geometry and materials comprising the $\mathrm{Si}_{3} \mathrm{~N}_{4}$ tip will not change significantly, the thermal resistance of tip, $R_{t}$, is regarded as a constant value.

In order to obtain a fuller understanding of the thermal profile generated by scanning the $\mathrm{SiN}_{\mathrm{x}}$ and gold wire, the thermal resistance of each tip-sample interaction can be estimated. As both the sample and probe have features with dimensions comparable to the mean free path of heat carriers $(\lambda)$, the effect of heat transfer in the ballistic regime must be considered. Thermal spreading resistance including both diffusive and ballistic heat transport can be calculated using $^{56}$

$$
R_{s p}=\frac{1}{4 k b_{c}}\left(1+\frac{8}{3 \pi} K_{n}\right),
$$

where $k$ is the thermal conductivity of the material, $b_{c}$ is the contact radius, and $K_{n}$ is the Knudsen number defined as $\lambda / b_{c}$. Sultan et al. ${ }^{57}$ have shown that the mean free path of carriers in $\mathrm{SiN}_{\mathrm{x}}$ is smaller than $2 \mathrm{~nm}$ at room temperature. It has been demonstrated that the contact radius between probes of the same tip geometry as those used in this work and a sample is $\sim 50 \mathrm{~nm} .{ }^{34}$ Therefore, thermal transport can be regarded, in this instance, as entirely diffusive due to the small Knudsen number. Furthermore, the $\mathrm{SiN}_{\mathrm{x}}$ film thickness of $400 \mathrm{~nm}$ makes it unnecessary to consider boundary scattering effects. Thus, the thermal spreading resistance of the $\mathrm{SiNx}$ can be given $\mathrm{by}^{28,34}$

$$
R_{\mathrm{SiN}}=\frac{1}{4 k_{\mathrm{SiN}} b_{c}},
$$

where $k_{\mathrm{SiN}}$ is the thermal conductivity of the $\operatorname{SiN}_{\mathrm{x}}[\sim 3 \mathrm{~W} / \mathrm{m}$ $\mathrm{K}$ (Ref. 57)]. This gives a value for $R_{\mathrm{SiN}}$ in the order of $10^{7} \mathrm{~K} / \mathrm{W}$. For the gold, the mean free path at room temperature is $10-30 \mathrm{~nm}$, dependent on whether the gold is single crystal or polycrystalline. ${ }^{58}$ Therefore, both diffusive and ballistic transport must be considered. Moreover, as the thickness of the gold is $20 \mathrm{~nm}$, substrate $\left(\mathrm{SiN}_{\mathrm{x}}\right)$ effects need to be considered when calculating the thermal spreading resistance. Accounting for all of these factors, thermal spreading resistance can be calculated using ${ }^{56,59}$ 

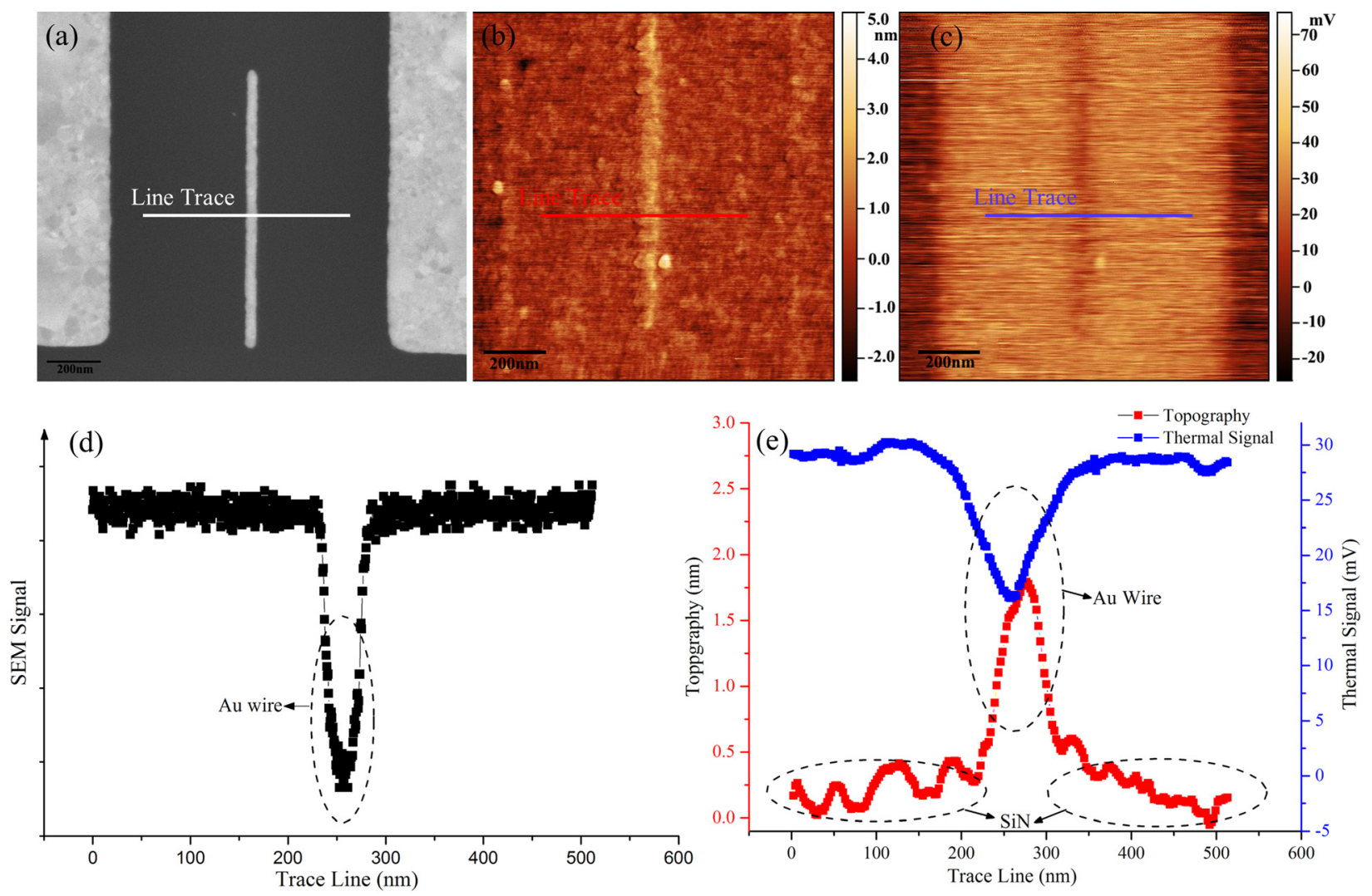

FIG. 7. (Color online) (a) Backscatter SEM image shows the $35 \mathrm{~nm}$ narrow gold wire, (b) AFM topographic image shows the same wire as (a), (c) thermal image of the same feature scanned by SThM probe, (d) plot of the line trace for SEM averaged over 16 lines, and (e) topography and thermal signals averaged over 16 lines. Dotted circles in (d) and (e) indicate the signal of the gold wire and $\mathrm{SiN}_{\mathrm{x}}$.

$$
R_{\mathrm{Au}}=\frac{1}{4 k_{\mathrm{Au}} b_{c K_{n}}}-\frac{1}{2 \pi k_{\mathrm{Au}} t} \log _{10}\left(\frac{2}{1+\frac{k_{\mathrm{Au}}}{k_{\mathrm{SiN}}}}\right),
$$

where $k_{\mathrm{Au}}$ and $t$ are thermal conductivity and thickness of the gold. This gives a thermal resistance for the thin film gold as being around $10^{4} \mathrm{~K} / \mathrm{W}$, which is 3 orders of magnitude smaller than that of $\mathrm{SiN}_{\mathrm{x}}$. We can infer from this that a dominant factor affecting the probe temperature is variation in sample spreading resistance. However, it is important to also consider other mechanisms.

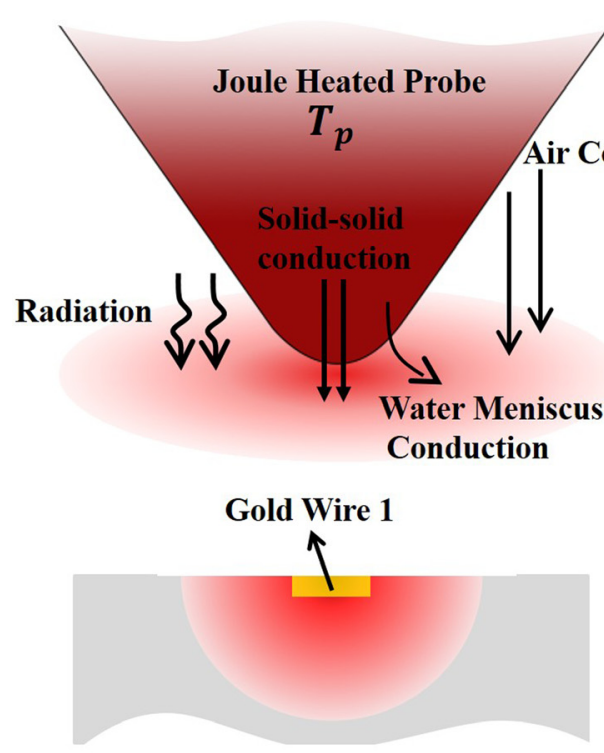

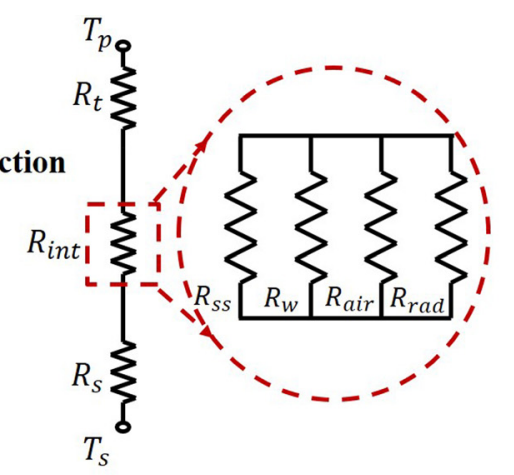

(a)

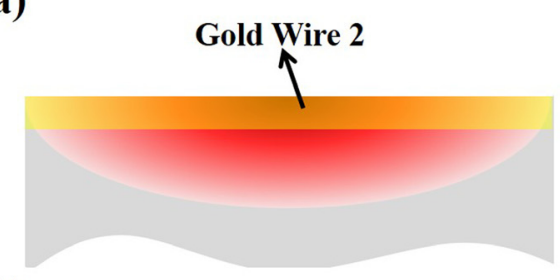

(b)

FIG. 8. (Color online) (a) Heat transfer interactions between the probe and the sample, together with the thermal resistance network showing the relationship between each interaction, and (b) different thermal diffusion as a result of variation in the width of the gold wires. Gold wire 1 and gold wire 2 indicate the narrow and wide wire, respectively. The heat spreading is represented by the hemispherical areas surrounding the gold wires. 
It has been recognized that the thermal resistance associated with heat transfer through the water meniscus is at least an order larger than that through the solid-solid contact and air conduction. ${ }^{27,31}$ This is in agreement with Assy and Gomès $^{32}$ who showed that the water meniscus radius for probes of the same tip geometry as that in this work at a similar temperature is smaller than $9 \mathrm{~nm}$, representing just $1 \%-6 \%$ of the total contact thermal conductance. This strongly suggests that the temperature of the tip as it scans across the sample is not dominated by the water meniscus.

Furthermore, the thermal interface resistance between the probe and the sample can be described as ${ }^{60,61}$

$$
R_{s s}=\frac{R_{B}}{\pi b_{c}^{2}},
$$

where $R_{B}$ is the thermal boundary resistance, having the same unit as the bulk thermal contact resistance. It has been recognized that, under ambient conditions, $R_{B}$ does not change significantly with sample materials and has a typical value of $1 \times 10^{-9}$ to $5 \times 10^{-8} \mathrm{~m}^{2} \mathrm{~K} / \mathrm{W} .^{28}$ For the hard materials (i.e., those that do not show plastic damage on scanning) used in this work, solid-solid contact radii $\left(b_{c}\right)$ do not vary greatly, and have been proved to be approximately $50 \mathrm{~nm}^{33,34}$ Therefore, it can be expected that the solid to solid interface thermal resistance, $R_{s s}$, will lie in the range of $10^{5}-10^{6} \mathrm{~K} / \mathrm{W}$.

Heat conduction through the air is another interaction that will affect the probe temperature. As the heat coefficient defined for the air gap between the tip and sample is independent of the sample material, ${ }^{56,62}$ the thermal resistance of the air gap will not vary with $\mathrm{SiN}_{\mathrm{x}}$ or gold and is considered to be on the order of $10^{5} \mathrm{~K} / \mathrm{W} .^{49}$ Therefore, when comparing all of these interactions between the tip and sample, the spatial variation of thermal spreading resistance due to different sample materials is the most likely candidate for causing the variations observed in the SThM thermal image.

As shown in Fig. 8(b), for the gold wire with small width ("Gold Wire 1"), heat spread into the sample will be largely controlled by the $\mathrm{SiN}_{\mathrm{x}}$, resulting in approximately cylindrical thermal diffusion. With an increase in the gold wire's width ("Gold Wire 2"), there is more heat spreading within the gold wire, due to the relatively high thermal conductivity of the gold, resulting in a lower probe temperature. At the limit of large wire width, the thermal signal measured from the gold wires is expected to tend toward to the same value observed when in contact with the gold pads, which may be considered to constitute an infinite in-plane feature.

The scan of a $75 \mathrm{~nm}$-width gold wire (Fig. 9) is also consistent with this explanation. The thermal signal shown in Fig. 9 is obviously larger when compared to that of the $35 \mathrm{~nm}$ wire (Fig. 7). Specifically, the difference in the thermal signal between the gold wire and the $\mathrm{SiN}_{\mathrm{x}}$ are $11.3 \mathrm{mV}$ for the $35 \mathrm{~nm}$ wire and $19.8 \mathrm{mV}$ for the $75 \mathrm{~nm}$ wire. The large effect of topographic artefacts is also seen in Figs. 9(b) and 9 (c) where particles (indicated by the circles $\mathrm{A} / \mathrm{A}^{\prime}$ and $\left.\mathrm{B} / \mathrm{B}^{\prime}\right)$ are observed to give rise to large thermal contrast.
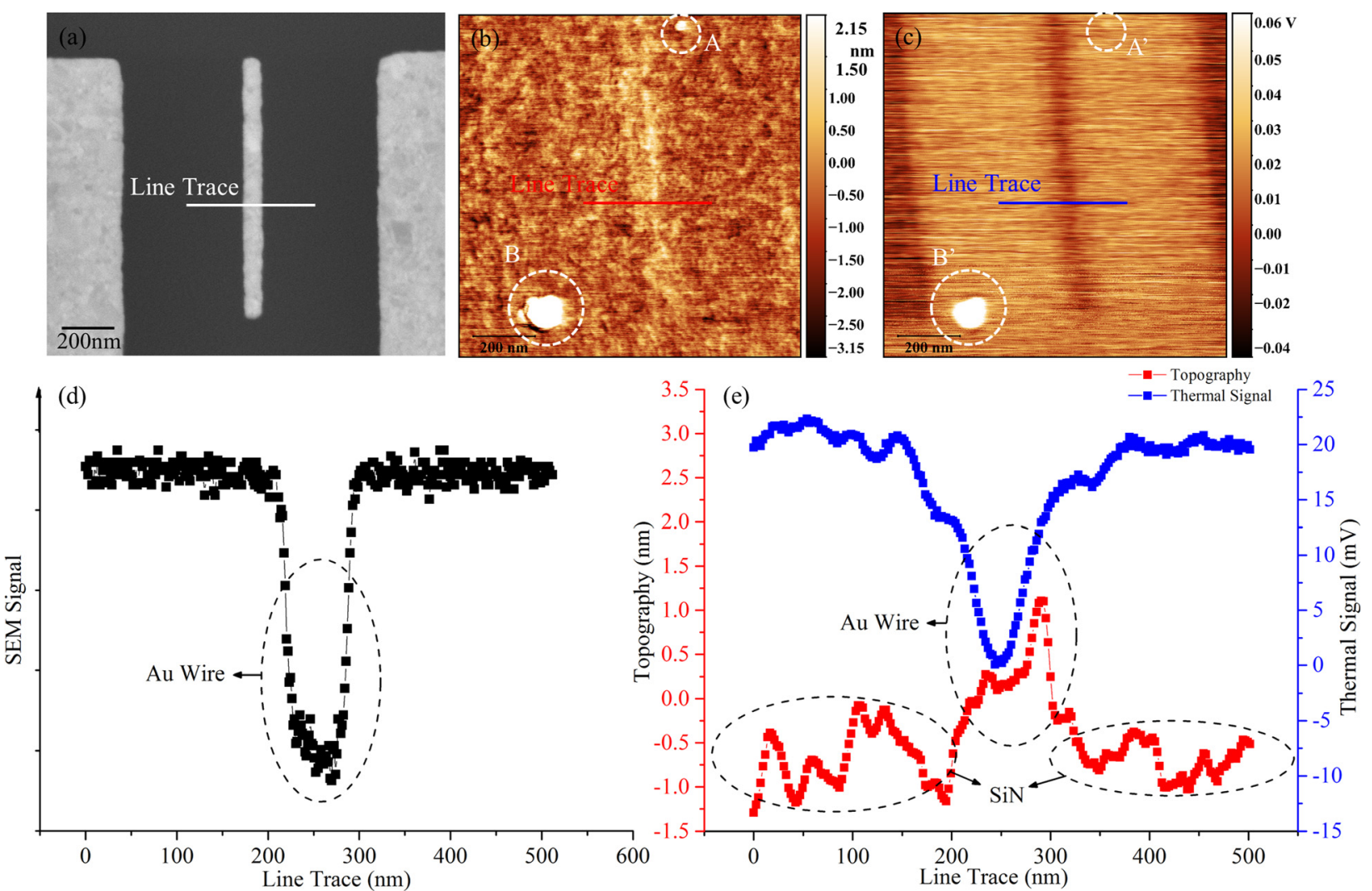

FIG. 9. (Color online) (a) SEM image shows the $75 \mathrm{~nm}$ narrow gold wire, (b) AFM topographic image, (c) thermal image shows the same feature scanned by SThM probe, and plots of the line traces from (d) SEM averaged over 16 lines and (e) topography and thermal signals averaged over 16 lines. Dotted circles in (d) and (e) indicate the signal of the gold wire and $\mathrm{SiN}_{\mathrm{x}}$. 
It should be noted that there is a small amount of topography associated with the gold features. However, this is of the same magnitude as the general roughness of the background $\mathrm{SiN}_{\mathrm{x}}$ and gold $(\sim 1 \mathrm{~nm})$. Therefore, any topographically induced artifacts in the thermal image are expected to be of the same size as the background noise present in the thermal signal. Any effect will certainly be much smaller than that present in an equivalent sample produced without using the method described in this work.

In order to easily visualize the change in thermal signal intensity, wires with widths ranging from 35 to $75 \mathrm{~nm}$ were scanned using the same experimental setup and the thermal signal from different pattern regions analyzed using a simple ratio, defined as

$$
\text { Ratio }=\frac{V_{\text {wire }}-V_{\mathrm{SiN}}}{V_{\text {pad }}-V_{\mathrm{SiN}}}
$$

where $V_{\text {wire }}, V_{\text {pad }}$, and $V_{\mathrm{SiN}}$ represent the thermal signal obtained on the gold wires, gold pads, and $\mathrm{SiN}_{\mathrm{x}}$, respectively. This ratio can be used to easily assess the relative signal magnitude as observed on wires of differing width scaled to that observed on gold pads. As the thermal signal on the wire approaches that of the background $\mathrm{SiN}_{\mathrm{x}}$, the ratio will approach 0 , whereas a ratio of 1 indicates the same signal as that observed on the large Au pads. It can be said that the closer the ratio is to 1 , the more "resolved" the Au wire is, that is, the signal becomes increasingly characteristic of the material, not the geometry. Figure 10 shows a plot of the ratio versus the width of the gold wires from 35 to $75 \mathrm{~nm}$. Values for $V_{\text {wire }}, V_{\text {pad }}$, and $V_{\mathrm{SiN}}$ were obtained from the SThM images by averaging a rectangular $5 \times 50$-pixel region on top of each feature as shown in Fig. 10 inset. The error bars represent \pm 1 standard deviation of the averaged values. As expected, the ratio observed on the gold wire slowly increases with an increase in the wire's width, simultaneously the standard deviation decreases due to the improved SNR associated with a larger signal. This shows that by employing a microfabricated SThM probe to scan a sample consisting two materials having a large difference in thermal

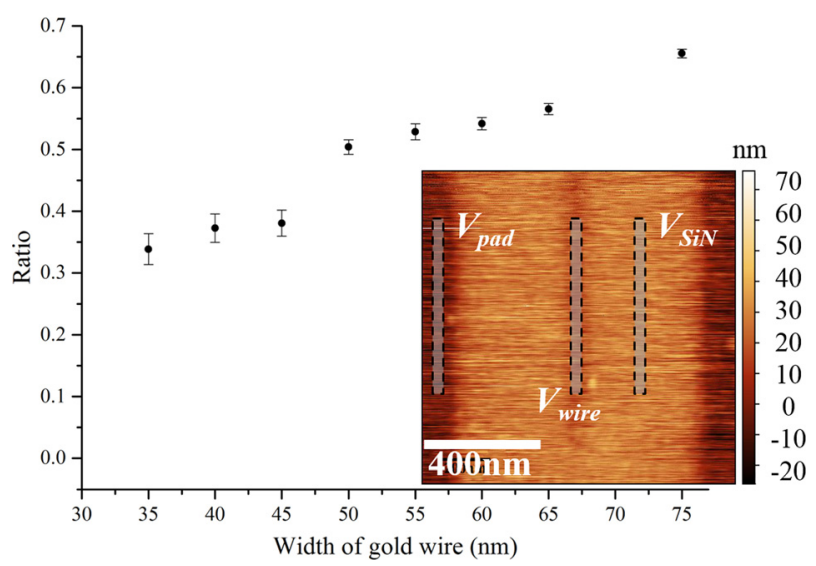

FIG. 10. (Color online) Plot of the thermal intensity ratio vs the width of the gold wire. The inset image shows the $5 \times 50$ pixels rectangular regions used to obtain the averaged signal from wire, gold pad and $\mathrm{SiN}_{\mathrm{x}}$. conductivity ( $\mathrm{SiN}_{\mathrm{x}}$ and $\mathrm{Au}$ in this case), the variation of pure thermal materials contrast can be quantified as a function of the size of the object being imaged. Hence, when scanning an object which is large, the contrast will have a limiting value, which is characteristic of the materials imaged. In contrast, for small objects, the contrast diminishes in a way which is in some sense characteristic of the material and the effective size of the SThM probe. By measuring this variation as a function of sample size, a robust and reproducible measure of the characteristic length scale which determines a SThM measurement of thermal conductivity may be obtained. In the present case we observe a smooth degradation of contrast for length scales down to $35 \mathrm{~nm}$, such that the observed signal at $75 \mathrm{~nm}$ scale has a contrast level which is $70 \%$ of that measured for large area films. This number is consistent with published claims of resolution for SThM using microfabricated probes.

\section{CONCLUSION}

We have demonstrated a general procedure to achieve lithographically defined, topography-free samples with significant materials contrast. The fabrication of the sample uses the commonly available fabrication methods of ebeam lithography, metal evaporation and liftoff, dielectric layer deposition, and wet etching. The utilization of multiple layers provides a stable and robust substrate. The method may be readily extended to allow the production of more complex samples with multiple materials and higher resolution patterns. It is also compatible with photolithographic pattern definition, allowing the low cost production of calibration samples.

The utility of these samples has been illustrated using SThM to generate high-resolution images, free of topographically induced artifacts. This data allowed the in-air thermalspatial response of a micromachined SThM probe to pure materials contrast to be unambiguously determined at length scales in the range of $35-70 \mathrm{~nm}$.

\section{ACKNOWLEDGMENTS}

The authors thank the QUANTIHEAT European Project (Grant Agreement No. 604668) for its financial support and the James Watt Nanofabrication Centre (JWNC) for access to facilities. The help of Donald MacLaren for providing access to the AFM is gratefully acknowledged.

${ }^{1}$ L. Zhang, T. Sakai, N. Sakuma, T. Ono, and K. Nakayama, Appl. Phys. Lett. 75, 3527 (1999).

${ }^{2}$ U. Hartmann, Annu. Rev. Mater. Sci. 29, 53 (1999).

${ }^{3}$ M. Nonnenmacher and H. K. Wickramasinghe, Appl. Phys. Lett. 61, 168 (1992).

${ }^{4} \mathrm{P}$. Klapetek, Quantitative Data Processing in Scanning Probe Microscopy: SPM Applications for Nanometrology (Elsevier, Burlington, 2012), p. 221, 245

${ }^{5}$ J. W. P. Hsu, Mater. Sci. Eng. 33, 1 (2001).

${ }^{6}$ V. F. Dryakhlushin, A. Y. Klimov, V. V. Rogov, and N. V. Vostokov, Appl. Surf. Sci. 248, 200 (2005).

${ }^{7}$ O. Fenwick, G. Latini, and F. Cacialli, Synth. Met. 147, 171 (2004).

${ }^{8}$ R. Mahajan, C. P. Chiu, and G. Chrysler, Proc. IEEE 94, 1476 (2006).

${ }^{9}$ S. Gradečak, F. Qian, Y. Li, H. G. Park, and C. M. Lieber, Appl. Phys. Lett. 87, 173111 (2005). 
${ }^{10}$ C. Guthy, C. Y. Nam, and J. E. Fischer, J. Appl. Phys. 103, 064319 (2008).

${ }^{11}$ J. Zou, J. Appl. Phys. 108, 034324 (2010).

${ }^{12}$ M. Kuball, S. Rajasingam, A. Sarua, M. J. Uren, T. Martin, B. T. Hughes, K. P. Hilton, and R. S. Balmer, Appl. Phys. Lett. 82, 124 (2003).

${ }^{13}$ D. Teyssieux, L. Thiery, and B. Cretin, Rev. Sci. Instrum. 78, 034902 (2007).

${ }^{14}$ T. Fujii, Y. Taguchi, T. Saiki, and Y. Nagasaka, Rev. Sci. Instrum. 83, 124901 (2012).

${ }^{15}$ C. C. Williams and H. K. Wickramasinghe, Appl. Phys. Lett. 49, 1587 (1986).

${ }^{16}$ L. Shi, O. Kwon, A. C. Miner, and A. Majumdar, J. Microelectromech. Syst. 10, 370 (2001).

${ }^{17}$ G. Mills, H. Zhou, A. Midha, L. Donaldson, and J. M. R. Weaver, Appl. Phys. Lett. 72, 2900 (1998).

${ }^{18}$ D. G. Cahill, H. E. Fischer, T. Klitsner, E. T. Swartz, and R. O. Pohl, J. Vac. Sci. Technol. A 7, 1259 (1989).

${ }^{19}$ S. Volz, X. Feng, C. Fuentes, P. Guérin, and M. Jaouen, Int. J. Thermophys. 23, 1645 (2002).

${ }^{20}$ V. M. Asnin, F. H. Pollak, J. Ramer, M. Schurman, and I. Ferguson, Appl. Phys. Lett. 75, 1240 (1999).

${ }^{21}$ A. Soudi, R. D. Dawson, and Y. Gu, ACS Nano 5, 255 (2011).

${ }^{22}$ F. Menges, H. Riel, A. Stemmer, and B. Gotsmann, Nano Lett. 12, 596 (2012).

${ }^{23}$ S. Somnath and W. P. King, Nanotechnology 25, 365501 (2014).

${ }^{24}$ K. Kim, J. Chung, G. Hwang, O. Kwon, and J. S. Lee, ACS Nano 5, 8700 (2011).

${ }^{25}$ A. Majumdar, Annu. Rev. Mater. Sci. 29, 505 (1999).

${ }^{26} \mathrm{~L}$. Shi, O. Kwon, G. Wu, and A. Majumdar, in Proceedings of the 38th Annual 2000 IEEE International Reliability Physics Symposium, San Jose, CA, 10-13 April 2000 (IEEE, Piscataway, NJ, 2000), pp. 394-398.

${ }^{27}$ L. Shi and A. Majumdar, J. Heat Transfer 124, 329 (2002).

${ }^{28}$ S. Gomès, A. Assy, and P. O. Chapuis, Phys. Status Solidi 212, 477 (2015).

${ }^{29}$ B. Gotsmann and M. A. Lantz, Nat. Mater. 12, 59 (2012).

${ }^{30}$ P. Klapetek, I. Ohlídal, and J. Buršík, Surf. Interface Anal. 38, 383 (2006).

${ }^{31}$ A. Assy, S. Lefèvre, P. O. Chapuis, and S. Gomès, J. Phys. D: Appl. Phys. 47, 442001 (2014).

${ }^{32}$ A. Assy and S. Gomès, Nanotechnology 26, 355401 (2015).

${ }^{33}$ M. Thompson Pettes and L. Shi, J. Heat Transfer 136, 032401 (2013).

${ }^{34}$ A. Assy and S. Gomès, Appl. Phys. Lett. 107, 043105 (2015).

${ }^{35}$ A. Hammiche, H. M. Pollock, M. Song, and D. J. Hourston, Meas. Sci. Technol. 7, 142 (1999).

${ }^{36}$ L. Zhou, G. Q. Xu, H. T. Ng, and S. F. Y. Li, J. Vac. Sci. Technol. B 15, 1871 (1997).

${ }^{37}$ S. Sadat, A. Tan, Y. J. Chua, and P. Reddy, Nano Lett. 10, 2613 (2010).

${ }^{38}$ L. Shi, S. Plyasunov, A. Bachtold, P. L. McEuen, and A. Majumdar, Appl. Phys. Lett. 77, 4295 (2000).
${ }^{39}$ M. Hinz, O. Marti, B. Gotsmann, M. A. Lantz, and U. Dürig, Appl. Phys. Lett. 92, 043122 (2008).

${ }^{40} \mathrm{G}$. Banerjee and R. L. Rhoades, ECS Trans. 13, 1 (2008).

${ }^{41}$ O. V. Kolosov, I. Grishin, and R. Jones, Nanotechnology 22, 185702 (2011).

${ }^{42}$ K. E. Docherty, S. Thoms, P. S. Dobson, and J. M. R. Weaver, Microelectron. Eng. 85, 761 (2008).

${ }^{43}$ J. J. Brophy, M. Epstein, and S. L. Webb, Rev. Sci. Instrum. 36, 1803 (1965).

${ }^{44}$ H. Zhou, K. Elgaid, C. Wilkinson, and I. Thayne, Jpn. J. Appl. Phys. 45, 8388 (2006).

${ }^{45}$ M. V. Lebedev, E. Mankel, T. Mayer, and W. Jaegermann, J. Phys. Chem. C 112, 18510 (2008).

${ }^{46}$ H. Zhou, A. Midha, G. Mills, S. Thoms, S. K. Murad, and J. M. R. Weaver, J. Vac. Sci. Technol. B 16, 54 (1998).

${ }^{47}$ B. E. E. Kastenmeier, P. J. Matsuo, J. J. Beulens, and G. S. Oehrlein, J. Vac. Sci. Technol. A 14, 2802 (1996).

${ }^{48}$ K. R. Williams, K. Gupta, and M. Wasilik, J. Microelectromech. Syst. 12, 761 (2003).

${ }^{49}$ P. C. Fletcher, B. Lee, and W. P. King, Nanotechnology 23, 035401 (2011).

${ }^{50}$ Y. Zhang, P. S. Dobson, and J. M. R. Weaver, J. Vac. Sci. Technol. B 30, 010601 (2012).

${ }^{51}$ S. Thoms and D. S. MacIntyre, J. Vac. Sci. Technol. B 28, C6H6 (2010).

${ }^{52}$ K. Luo, Z. Shi, J. Varesi, and A. Majumdar, J. Vac. Sci. Technol. B 15, 349 (1997).

${ }^{53}$ L. Shi and A. Majumdar, Applied Scanning Probe Methods Part II (Springer, Berlin, Heidelberg, 2004), pp. 327-362.

${ }^{54}$ V. V. Gorbunov, N. Fuchigami, J. L. Hazel, and V. V. Tsukruk, Langmuir 15, 8340 (1999).

${ }^{55}$ W. P. King, B. Bhatia, J. R. Felts, H. J. Kim, B. Kwon, B. Lee, S. Somnath, and M. Rosenberger, Annu. Rev. Heat Transfer 16, 287 (2013).

${ }^{56}$ R. Prasher, Nano Lett. 5, 2155 (2005).

${ }^{57}$ R. Sultan, A. D. Avery, J. M. Underwood, S. J. Mason, D. Bassett, and B. L. Zink, Phys. Rev. B 87, 214305 (2013).

${ }^{58}$ D. Canchal-Arias and P. Dawson, Surf. Sci. 577, 95 (2005).

${ }^{59}$ M. M. Yovanovich, J. R. Culham, and P. Teertstra, IEEE Trans. Compon. Packag. A 21, 168 (1998).

${ }^{60}$ B. Gotsmann, M. A. Lantz, A. Knoll, and U. Dürig, "Nanoscale thermal and mechanical interactions studied using heatable probes," in Nanotechnology, edited by H. Fuchs (Wiley-VCH, Weinheim, 2009), Vol. 6, pp. 121-169.

${ }^{61}$ B. A. Nelson and W. P. King, Nanoscale Microscale Thermophys. Eng. 12, 98 (2008).

${ }^{62}$ V. Bahadur, J. Xu, Y. Liu, and T. S. Fisher, J. Heat Transfer 127, 664 (2005). 